\title{
Investigations on a hybrid element with cellular metallic material for heating, cooling and ventilation
}

\author{
Lars Schinke ${ }^{1, *}$, André Schlott ${ }^{2}$, Maximilian Beyer ${ }^{1}$, Joachim Seifert ${ }^{1}$, and Marcel Fink ${ }^{2}$ \\ ${ }^{1}$ Technische Universität Dresden, Institute of Power Engineering, 01069 Dresden, Germany \\ ${ }^{2}$ Fraunhofer Institute for Manufacturing Technology and Advanced Materials IFAM, Branch Lab Dresden, 01277 Dresden, Germany
}

\begin{abstract}
Surface heating and cooling systems are gaining more and more importance in building energy technology. In order to implement these systems not only on the floor but also on the ceilings and walls, the thermodynamic requirements are supplemented by constructional requirements. In order to fulfil these requirements, the use of open-cell porous metals for such systems will be investigated in more detail. In addition to the design and manufacturing challenges, a large number of numerical and experimental investigations on test samples are also necessary. These investigations are described and explained in more detail in the following article.
\end{abstract}

\section{Introduction}

The use of surface heating systems will gain in importance in the future, [1]. On the one hand, the systems can be operated at lower system temperatures and thus represent an energy optimisation compared to convective heating systems. On the other hand, the user accepts such a system better with regard to thermal comfort.

A new solution is currently under investigation, especially for the use in renovation projects. This is a hybrid element, which combines the advantages of surface heating in the winter with cooling in the summer and all-season ventilation. In recent years, extensive research on open-cell porous metals (OCPM) has taken place in materials science. Examples are the metal foam and the metal fibres, $[2,3]$. These materials have various advantages for use in building energy technology, $[4,5]$. In the hybrid element a OCPM is used to distribute heat from the pipe to the room-side wall (or in summer from the wall to the pipe). In addition, there is a channel for conditioning the supply air temperature before entering the room. A concept can be seen in Fig. 1.

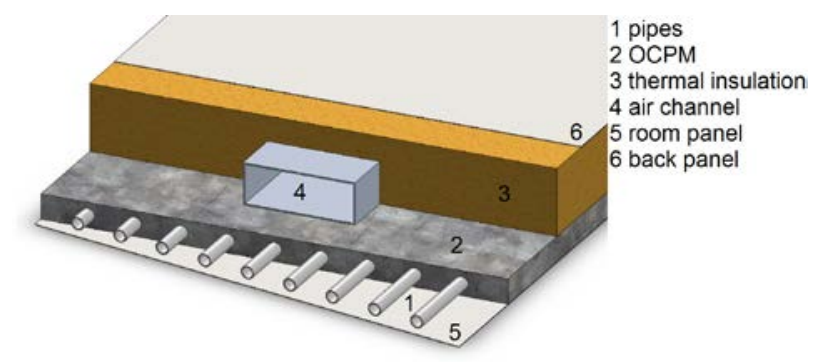

Fig. 1. Concept of a hybrid element

This gives the element the following advantageous properties:
- fulfilment of all HVAC tasks (Heating, Ventilation, Air Conditioning) incl. cooling

- $\quad$ lightweight and compact design

- very good heat distribution and thus reduction of thermal inertia compared to classic underfloor heating

- thus possibility to increase pipe distance at a nearly constant mean surface temperature

- $\quad$ very low surface temperature ripple

- variable installation options (ceiling or wall).

\section{Manufacturing of test samples}

In addition to thermodynamic and energetic considerations, the manufacturing of the hybrid element represents a major focus within ongoing investigations. Above all, the production of the composite of tube and OCPM is a challenge from a manufacturing point of view. The connection must be both friction-locked and form-fit to ensure good heat transfer.

For this reason, various manufacturing processes were applied and test samples with a size of $\mathrm{w} \times 1 \times \mathrm{h}=$ $200 \mathrm{~mm} \times 200 \mathrm{~mm} \times 20 \mathrm{~mm}$ were manufactured. In addition to the manufacturing processes, the test samples also differed with regard to the pipe material. The pipe materials used are copper, aluminium and polyethylene (PE).

A variation of the OCPM was also considered. Fig. 2 shows the different pipe materials in a metal foam test sample.

\footnotetext{
* Corresponding author: 1ars.schinke@tu-dresden.de
} 


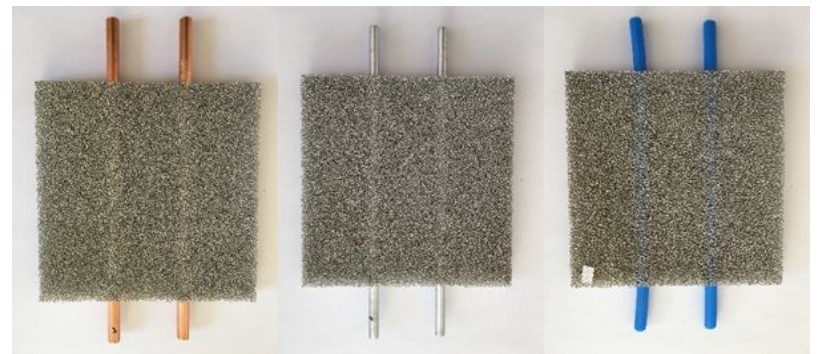

Fig. 2. Test samples of metal foam with pipes of copper, aluminium and $\mathrm{PE}$ (from left to right)

Fig. 3 shows a test sample of metal fibers with pipes of aluminium. Currently, both OCPM are investigated and analysed.

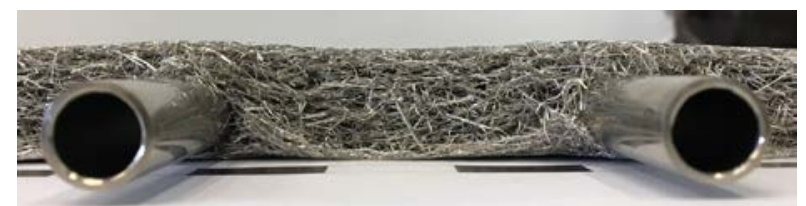

Fig. 3. Test samples of metal fibres with pipes of aluminium

\section{Thermal investigations}

Thermal investigations are carried out to determine the heating power and the temperature ripple on the surface. These examinations are split into a numerical and an experimental part. Both parts are needed to find the most powerful geometrical, material and operating parameters for the given application.

\subsection{Thermal simulation}

At first, the numerical simulations represent an idealized module setup without any thermal contact resistances between different parts like pipe, OCPM or the used surrounding sheet metal. Therefore, the influence of different geometrical parameters can be investigated with reasonable effort and time. This includes, among other things, the height of the OCPM, the pipe spacing as well as the pipe material and fluid temperature. Furthermore, the thermal properties of the different parts are varied in order to specify property limits for every component. Both investigated OCPM are simulated. With the simulation results, the needed directional dependent heat conductivity of the OCPM is evaluated and so the needed structure type and material porosity can be determined.

An exemplary 2D model used in this first step is shown in Fig. 4. It contains four pipes which are positioned in the centre of the OCPM (V1) and are laterally equally spaced. In a second model version, the pipes are set in the same lateral position and height regarding the room panel, but the OCPM height is reduced so the pipes are located on the connecting surface between OCPM and thermal insulation (V2). To maintain the overall height, the thermal insulation thickness is increased in model version V2. The insulation thickness in both models fulfil all regulations according to [6] when connected to an unheated wall of $10^{\circ} \mathrm{C}$ at the back panel. The heating fluid is considered as a constant temperature boundary condition of $40{ }^{\circ} \mathrm{C}$ inside all pipes. The heat transfer to the room is set as a free convection boundary on the bottom side of a heated plate, which results in an heat transfer coefficient of about $5 \mathrm{~W} /\left(\mathrm{m}^{2} \mathrm{~K}\right)$.

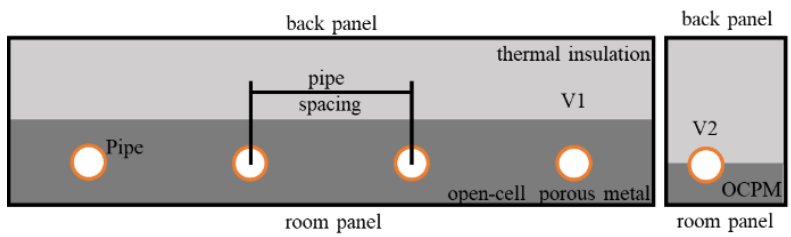

Fig. 4. 2D simulation model for principal investigations (left) and pipe position variations (right)

The result of the numerical investigations are the thermal behaviour of the room panel, especially the minimal, maximal and mean temperature as well as the heating power per surface area. The parameters are rated on their influence on the temperature and heating power and the design parameters are defined. Based on the results of these simulations, parameter combinations for the following measurements are defined. After defining the main impact parameters, the pipe spacing is optimized to reach a maximum ripple amplitude of $\Delta \mathrm{T}_{\mathrm{R}}$ of $\pm 0.5 \mathrm{~K}$, $\pm 1.0 \mathrm{~K}$ and $\pm 1.5 \mathrm{~K}$.

As a second step, the influence of the pipe position within the OCPM is investigated. At the beginning, the pipe were centred in the height of the OCPM. The impact of the pipe distance from the room panel and the OCPM height up to the point where the OCPM is not enclosing the pipe completely is analysed.

\subsection{Experimental investigations}

For thermodynamic and energetic analysis a basic experimental setup was developed, see Fig. 5. The test sample is connected to a thermostatic bath so that a water flow with a defined supply temperature $\left(\vartheta_{\mathrm{S}}\right)$ and volumetric flow rate $(\dot{V})$ is provided. In addition, the return temperature $\left(\vartheta_{R}\right)$ and ambient temperature $\left(\vartheta_{A}\right)$ are measured.

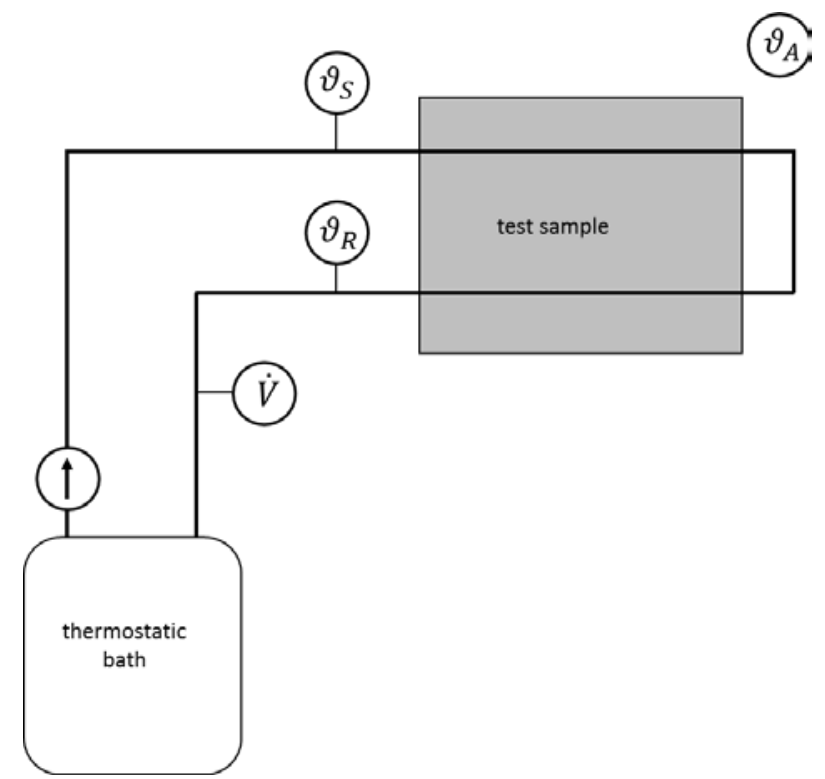

Fig. 5. Basic experimental test setup 
This allows the caloric energy balance of the water to be calculated with equation (1):

$$
\dot{Q}=\dot{V} \cdot \rho \cdot c_{\mathrm{p}} \cdot\left(\vartheta_{\mathrm{S}}-\vartheta_{\mathrm{R}}\right)
$$

The transferred heat flux Q represents an evaluation variable for the various test specimens examined.

The first experimental method based on the free convection is used to obtain information on the heat distribution in the composite of pipe and OCPM. This means that the test specimen lies on a thermal insulation and emits the heat to the environment by free convection on the free top side. Fig. 6 shows an example of the test setup for this investigation method.

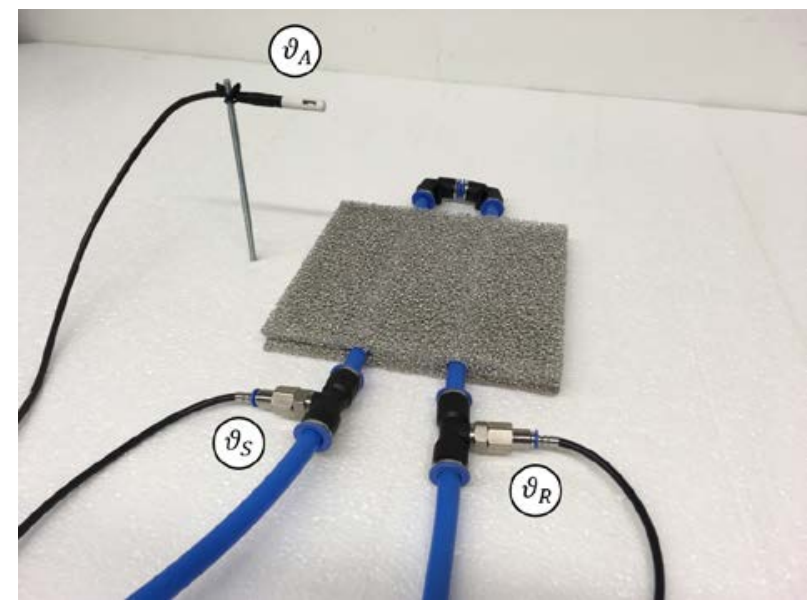

Fig. 6. Test setup for the free convection method

For the exact determination of the transferable heat flux it is necessary that defined boundary conditions are applied to the test sample. This is done by placing the test sample between two plates with an approximately constant, uniform surface temperature. This is achieved by a high water flow rate through the plates at a defined temperature. Thus, the heat is transferred symmetrically from the water in the tube via the OCPM to the plates. The specific heat flow can be measured with the aid of two heat flow measuring plates. In Fig. 7, the test setup for the method plate measuring can be seen. This second experimental method is based on $[7,8]$.

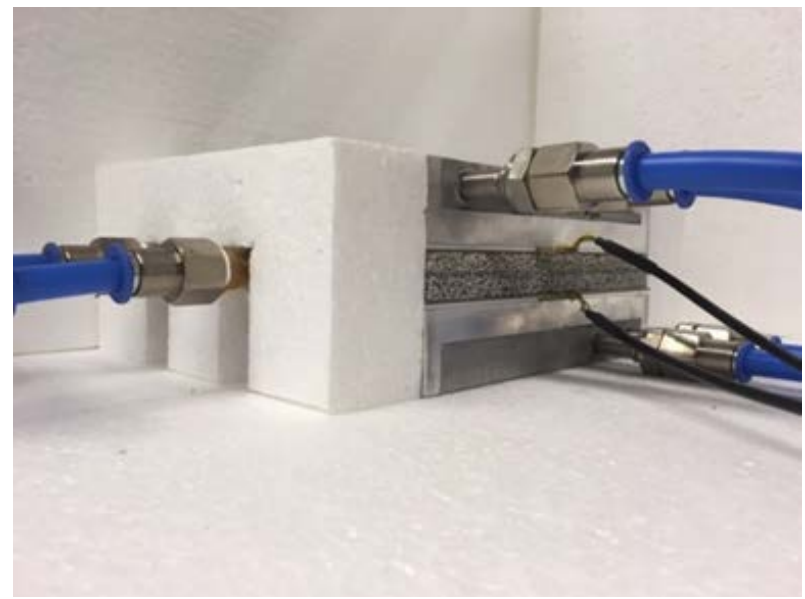

Fig. 7 .Test setup for the plate measuring method
Table 1 shows a summary of the two used measurement methods.

Table 1. Comparison of the measurement methods

\begin{tabular}{|c|l|l|}
\hline & Free convection & \multicolumn{1}{c|}{ Plate measuring } \\
\hline description & $\begin{array}{l}\text { - heat distribution } \\
\text { in the test sample } \\
\text { - dynamic effects }\end{array}$ & $\begin{array}{l}\text { - detailed performance } \\
\text { measurement } \\
\text { - detection of thermal } \\
\text { resistances }\end{array}$ \\
\hline $\begin{array}{c}\text { additional } \\
\text { sensors }\end{array}$ & $\begin{array}{l}\text { - thermography } \\
\text { - ambient } \\
\text { temperature }\end{array}$ & $\begin{array}{l}\text { - heat flow measuring } \\
\text { plate }\end{array}$ \\
\hline $\begin{array}{c}\text { measured } \\
\text { values }\end{array}$ & $\begin{array}{l}\text { - heat flux } \\
\text { - temperature } \\
\text { distribution }\end{array}$ & - specific heat flux \\
\hline
\end{tabular}

\section{Results}

The following section shows the results of the different investigations.

\subsection{Thermal Simulation}

Fig. 8 shows the optimal pipe spacing as a function of the maximum ripple amplitude. Curve parameter is the heat conductivity of the OCPM from 1.0 to $6.0 \mathrm{~W} /(\mathrm{m} \mathrm{K})$. The different line styles represent the two compared pipe positions within the OCPM, where V1 represents the pipe centred within the OCPM and V2 means the pipe centre line is located in the connection surface of the OCPM and the thermal insulation.

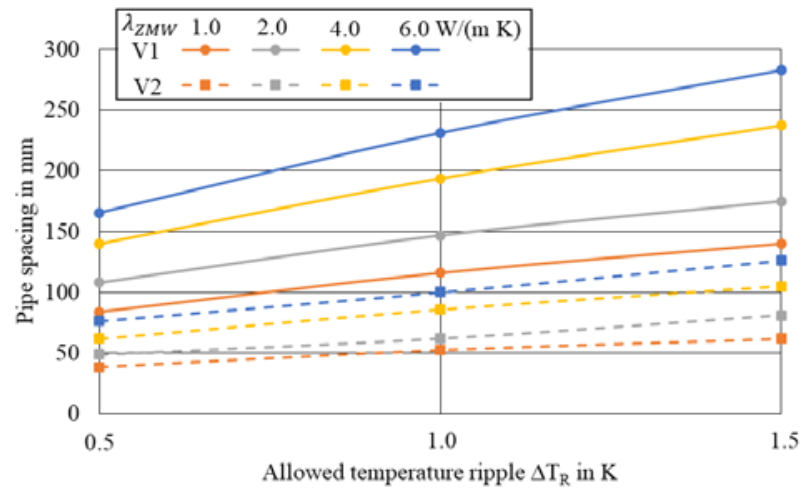

Fig. 8. Optimal pipe spacing as a function of temperature ripple amplitude and heat conductivity of the OCPM.

As shown, both the pipe positioning within the OCPM and the heat conductivity of the OCPM have great impact on the pipe spacing. As anticipated, the possible pipe spacing is increased with a higher heat conductivity of the OCPM. In addition, the centred position of the pipe within the OCPM results in higher possible pipe spacing. This is because the connection surface is doubled compared to the V2 position. With pipe position $\mathrm{V} 2$, the OCPM layer height can be reduced to compensate for the different distance between room plate and pipe centre line. This results in slightly higher pipe spacing possible, but the pipe position V1 is preferable. 
The influence of both parameters on the possible surface temperature is shown in Fig. 9.

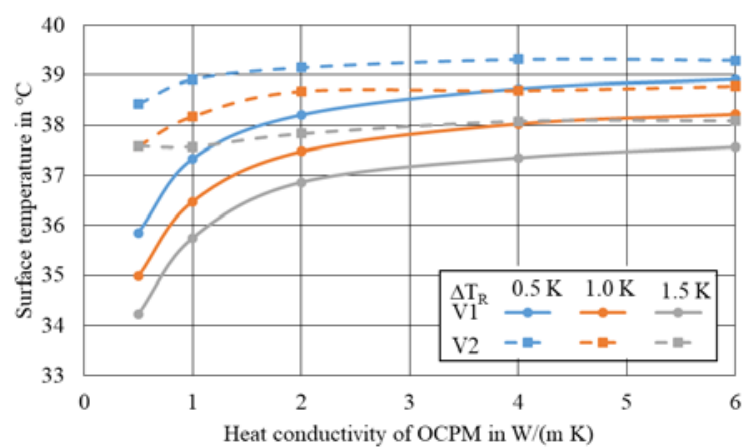

Fig. 9. Mean surface temperature as a function of heat conductivity of the OCPM and temperature ripple amplitude.

The lower pipe spacing of pipe position V2 shown in Fig. 8 results in a higher mean surface temperature compared to V1. In addition, a lower allowed temperature ripple results in a higher mean surface temperature. Both statements especially apply at lower OCPM heat conductivity. With higher heat conductivity of the OCPM, the results converge to the same level of surface temperature and are therefore equally suitable for the planned heating element.

The heating power per surface area is directly connected to the mean surface temperature. Therefore, the results presented in Fig. 10 show the same behaviour. With the given configuration, heating powers of about 80 to $95 \mathrm{~W} / \mathrm{m}^{2}$ can be achieved, depending on the thermal conductivity of the OCPM and the allowed temperature ripple at the surface.

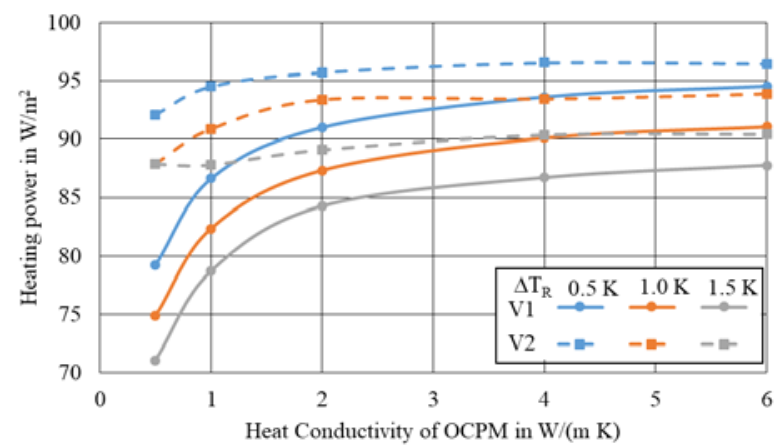

Fig. 10. Heating Power per surface unit as a function of heat conductivity of the OCPM and temperature ripple amplitude.

Fig. 11 and 12 show the temperature distribution within the model at different heat conductivities of the OCPM for model version $\mathrm{V} 1$ and $\mathrm{V} 2$, respectively. The temperature ripple is in both figures $\pm 0.5 \mathrm{~K}$. Due to the fixed number of pipes, the different pipe spacing results in different module sizes depending on the heat conductivity of the OCPM.

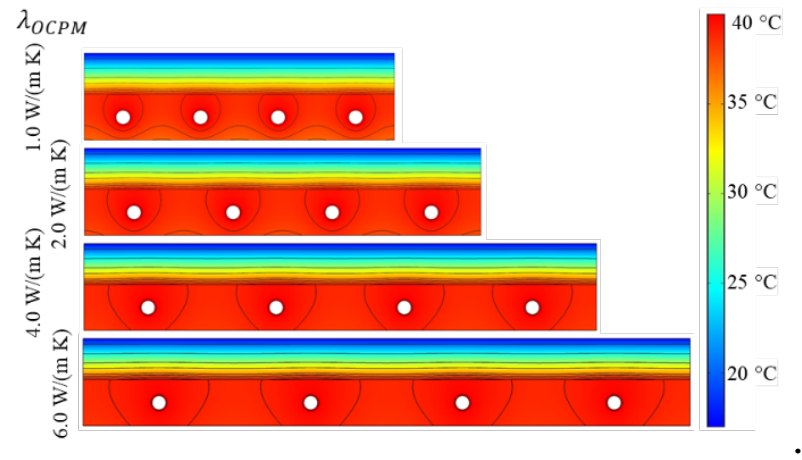

Fig. 11. Temperature distribution in model $\mathrm{V} 1$ at different OCPM heat conductivity with a temperature ripple of $\Delta T_{R}$ of $\pm 0.5 \mathrm{~K}$

The model height is true to scale, so that the length of each model in Fig. 11 and 12 can be compared and the lower pipe spacing in model V2 can be seen. Solid lines of constant temperature show that in V1 the range of different temperatures are more equally spaced than in V2 where the temperature lines come closer together between the pipes.

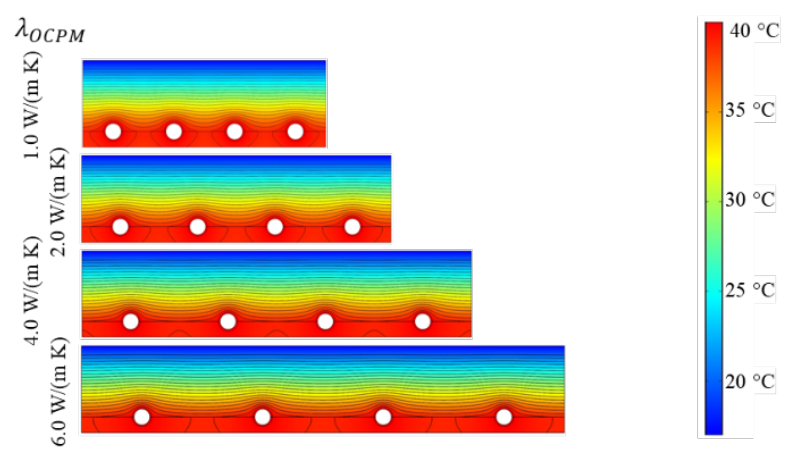

Fig. 12. Temperature distribution in model $V 2$ at different OCPM heat conductivity with a temperature ripple of $\Delta T_{R}$ of $\pm 0.5 \mathrm{~K}$

\subsection{Experimental Investigations}

For all test samples, the two methods described in section 3.2 were used for the experimental investigations. The supply temperature $\left(\vartheta_{\mathrm{S}}=30^{\circ} \mathrm{C}\right.$, $\left.40^{\circ} \mathrm{C}, 50^{\circ} \mathrm{C}\right)$ and the volumetric flow rate $(\dot{\mathrm{V}}=0.21 / \mathrm{min}$, $0.5 \mathrm{l} / \mathrm{min}, 2.5 \mathrm{l} / \mathrm{min}$ ) were varied.

The Fig. 13-15 show the temperature distribution on the test sample. The influence of the pipe material is clearly visible. The test samples with metallic pipes (especially copper, see Fig 13) have a good heat distribution due to the greater thermal conductivity. When PE is used as the pipe material (Fig. 15), a thermal hotspot is visible around the pipe, which is due to the different emission coefficient.

With the help of the free convection method, statements can also be made regarding the evolution of the temperature distribution over time. Here, too, the test samples with metallic pipes are heated to a stationary state faster than the test samples with PE pipes. 


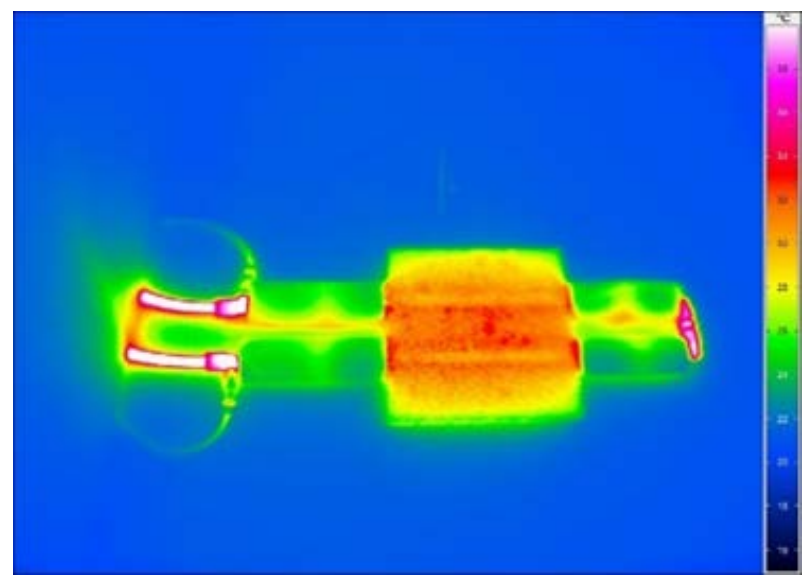

Fig. 13. Temperature distribution of the test sample with copper pipes at $\dot{V}=2.5 \mathrm{l} / \mathrm{min}$ and $\vartheta_{\mathrm{s}}=40{ }^{\circ} \mathrm{C}$

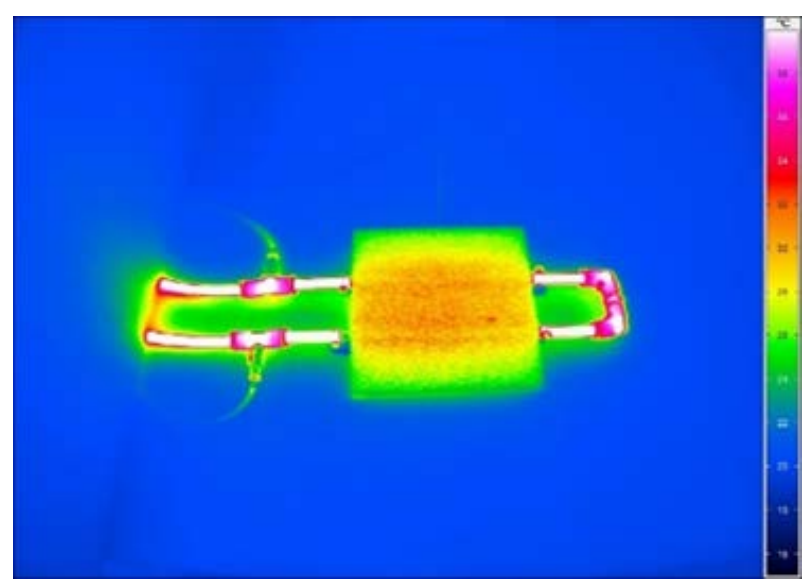

Fig. 14. Temperature distribution of the test sample with aluminium pipes at $\dot{\mathrm{V}}=2.5 \mathrm{l} / \mathrm{min}$ and $\vartheta_{\mathrm{S}}=40^{\circ} \mathrm{C}$

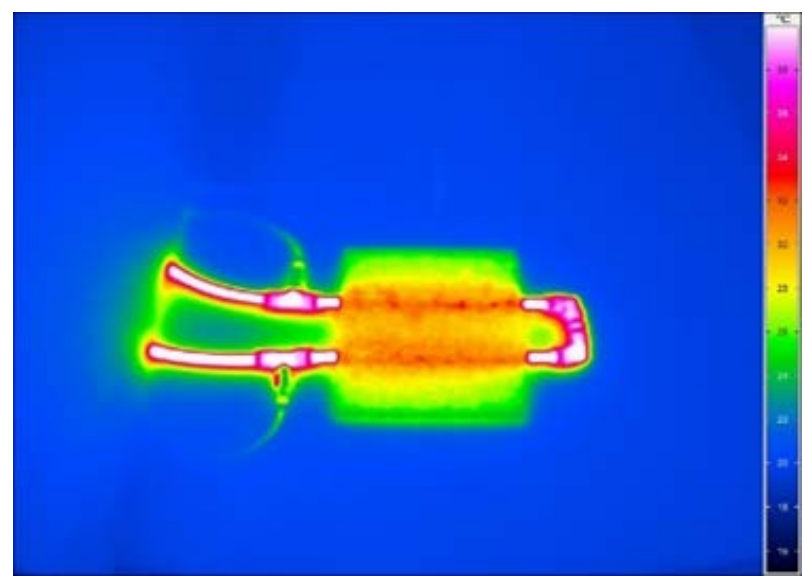

Fig. 15. Temperature distribution of the test sample with PE pipes at $\dot{\mathrm{V}}=2.5 \mathrm{1} / \mathrm{min}$ and $\vartheta_{\mathrm{S}}=40^{\circ} \mathrm{C}$

The influence of the manufacturing process on the composite of pipe and OCPM can also be seen. The manufacturing processes, which provide a good and large area thermal contact between pipe and OCPM provide, are expected to provide better thermal properties.

Extensive measurements were also carried out using the plate measuring method. The Fig. 16 shows an example of the specific heat flux at different volumetric flow rates and supply temperatures.
It can be seen that the specific heat flux increases with increasing volumetric flow rate. The supply temperature also has a major influence, which as expected results in a higher specific heat flux at high supply temperature values.

Furthermore, the influence of the pipe material is clearly visible. With pipes made of copper (Co), significantly higher specific heat fluxes can be transferred than with pipes made of PE.

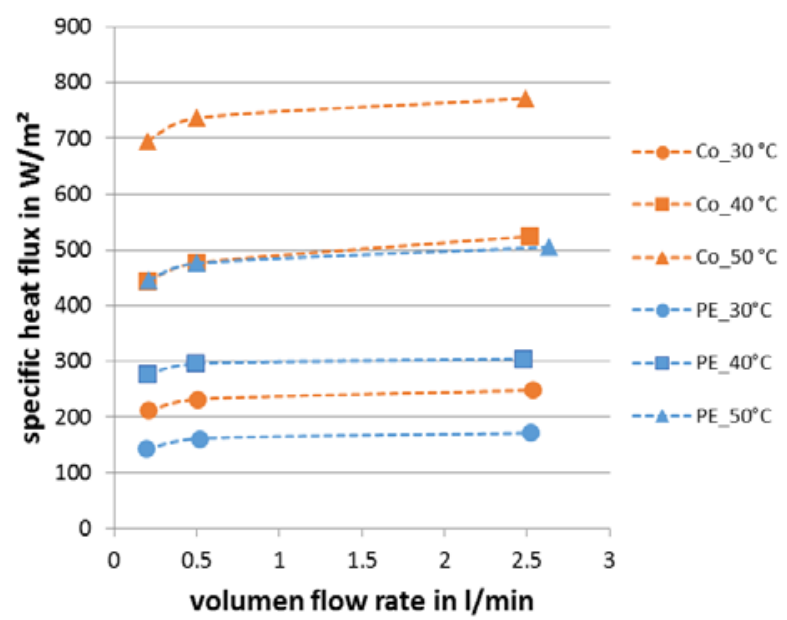

Fig. 16. Specific heat flux for different volumetric flow rates and supply temperatures

It should be noted that the specific heat flux achieved here applies only to the composite of pipe and OCPM. When used in a building, for example as ceiling heating or cooling, the heat flux is significantly reduced due to the additional thermal resistance, like contact resistances and the heat transfer to the room. Therefore, the heat flux values are reduced to values described in section 4.1.

\section{Summary and outlook}

The results presented in this article show that open porous metallic materials can be useful in building energy technology. The heat transport of a water-based system consisting of pipe, OCPM and room-side wall is very well feasible. With a suitable choice of pipe spacing, the temperature ripple can be significantly reduced and, according to current measurements, at least a specific heat flow of $95 \mathrm{~W} / \mathrm{m}^{2}$ can be achieved. This is comparable to market available ceiling heating systems. The next steps will include further investigations of the cellular metallic material. The type (metal foam, fibres) and porosity of the OCMP as well as the pipe distance from the room panel and the type of OCPM enclosing the pipe are the focal points. Afterwards, the system integration of the hybrid element into the building takes place, so that all requirements for heating, cooling and ventilation can be evaluated.

For now, both pipe positions investigated can reach the same requirements regarding the allowed temperature ripple. Reducing the OCPM saves expensive material and reduces the module weight. Additionally, the pipe position V2 increases the possible heating power. On the 
downside, more pipes are needed for a given element size because of the lower possible pipe spacing at a given temperature ripple at the room panel. This leads to an overall higher element weight.

With the described experimental setup in connection with the simulation model, further knowledge gains are possible. They are used to determine the thermal contact resistances between the different components as shown in Fig. 17. $R_{h 1}$ and $R_{h 2}$ represent the convective heat transfer within the pipe and from the panel to the room, respectively. $R_{c 1}$ and $R_{c 2}$ are the contact resistances within the element. For the determination of $R_{h 2}$ results of $[9,10,11]$ can be used.

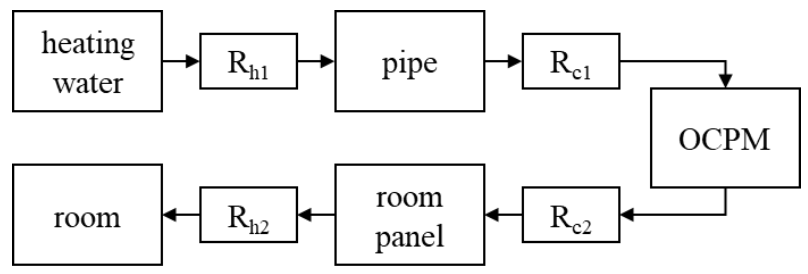

Fig. 17. Thermal resistances between the components of a hybrid element

To determine $R_{c 1}$ and $R_{c 2}$, the results of the thermal measurements described in section 4.2 are compared to the simulated values. By adjusting one relevant contact resistance at a time so that measured and simulated temperatures match, the value of this contact resistance is determined. This is repeated until all contact resistances are analysed.

With a 3D model currently in development, the fluid flow rate and the temperature drop within the heating pipes will be simulated. This allows to analyse different flow configurations in order to represent the optimal pipe arrangement for minimal temperature ripple at the room panel and low pressure drop within the hybrid element. The evaluated flow configurations also take installation requirements into account.

Acknowledgement: This research was supported by the German Federal Ministry for Economic Affairs and Energy under the project number 03ET1512A-B

\section{References}

1. S. Serrano, D. Ürge-Vorsatz, C. Barreneche, A. Palacios, L. F. Cabeza, Energy, 119, 425 (2017)

2. O. Andersen, C. Kostmann, G. Stephani, G. Korb, MetFoam (2003)

3. M. Fink, O. Andersen, T. Seidel, A. Schlott, Metals, 8, 554 (2018)

4. C.Y. Zhao, Int. J. Heat Mass Tansf., 55, 3618 (2012)

5. C. Veyhl, T. Fiedler, O. Andersen, J. Meinert, T. Bernthaler, I.V. Belova, G.E. Murch, Int. J. Heat Mass Transf., 55, 2440 (2012)

6. DIN EN 1264, Water based surface embedded heating and cooling systems (2011)
7. EN 12667, Thermal performance of building materials and products - Determination of thermal resistance by means of guarded hot plate and heat flow meter methods (2001)

8. ISO 8302, Thermal insulation - Determination of steady-state thermal resistance and related properties - Guarded hot plate apparatus (1991)

9. N. Fonseca Diaz, J. Lebrun, P. André, Int. J. Refrig., 33, 793 (2010)

10. F. Causone, S. P. Corgnati, M. Filippi, B. W. Olesen, Energ. Buildings, 41, 622 (2009)

11. M. Andrés-Chicote, A. Tejero-González, E. Velasco-Gómez, F. J. Rey-Martínez, Energ. Buildings, 54, 207 (2012) 